\title{
Nanoparticle-targeted photoacoustic cavitation for tissue imaging
}

\author{
James R. McLaughlan ${ }^{* a}$, Ronald A. Roy ${ }^{\mathrm{a}}$, Hengyi Ju ${ }^{\mathrm{b}}$ and Todd W. Murray ${ }^{\mathrm{b}}$ \\ ${ }^{a}$ Dept. of Mechanical Engineering, Boston Univ. \\ 110 Cummington St., Boston, MA 02215 USA \\ ${ }^{b}$ Dept. of Mechanical Engineering, Univ. of Colorado Boulder, \\ 427 UCB, Boulder, CO 80309 USA
}

\begin{abstract}
Photoacoustic tomography is a non-invasive imaging technique based on the detection of broadband acoustic emissions generated by the absorption of light in tissue. This technique utilises the high contrast of optical imaging with high resolution from ultrasound imaging. However, the ability to detect these emissions above the noise level ultimately limits the depth to which imaging can be performed. Introduction of light-absorbing gold nanoparticles can improve the signalto-noise ratio in tissue, through greater optical absorption and targeting specific cell populations, thereby enhancing contrast and the ability to delineate tissue types. For sufficiently high laser fluence incident on a nanoparticle, a transient vapour cavity is formed and undergoes inertial collapse, generating a broadband emission and possibly additional contrast. However, the laser fluence required to achieve this typically exceeds the maximum permissible exposure (MPE) for tissue. Through the combination of ultrasonic and optical pulses, the light and sound thresholds required to repeatedly generate inertial cavitation were reduced to $11.1 \mathrm{~mJ} / \mathrm{cm}^{2}$ and $1.5 \mathrm{MPa}$ respectively. Experiments employed a transparent acrylamide gel possessing a small $(<600 \mu \mathrm{m})$ spherical region doped with $80 \mathrm{~nm}$ diameter gold nanoparticles and simultaneously exposed to pulsed laser light $(532 \mathrm{~nm})$ and pulsed ultrasound $(1.1 \mathrm{MHz})$. The amplitude of broadband emissions induced by both light and sound exceeded that produced by light alone by almost two orders of magnitude, thereby facilitating imaging a deeper depth within tissue. 2D images of doped regions generated from conventional photoacoustic and ultrasound-enhanced emissions are presented and compared.
\end{abstract}

Keywords: Photoacoustic effect, gold nanoparticles, inertial cavitation, high intensity focused ultrasound, passive cavitation detection, vapourisation, tissue imaging.

\section{INTRODUCTION}

The generation of broadband ultrasonic waves from the thermoelastic expansion of a light-absorbing medium is of significant interest for imaging applications ${ }^{1}$. These photoacoustic (PA) emissions are typically generated through the illumination of short (ns) pulses of laser light ${ }^{2}$, where the pulse length is less than the acoustic and thermal relaxation times of the medium. The pressure amplitude of the PA emissions is proportional to the incident laser fluence, where differences in optical absorption can provide contrast between tissue types.

*jmcl@bu.edu; phone 1617-353-7381

Photons Plus Ultrasound: Imaging and Sensing 2010, edited by Alexander A. Oraevsky, Lihong V. Wang, Proc. of SPIE Vol. 7564, 756415 - (c) 2010 SPIE · CCC code: 1605-7422/10/\$18 · doi: 10.1117/12.842415 
Detection of PA emissions form the basis of photoacoustic tomography ${ }^{3}$ (PAT) and microscopy ${ }^{4}$ (PAM), which have been used for high resolution imaging of skin, breast tissue and small animals ${ }^{1}$. These imaging techniques have the advantage of combining high spatial resolution of ultrasound imaging with the high contrast of optical imaging. The signal-to-noise ratio of the detected PA emissions governs the contrast of this imaging technique ${ }^{5}$. This ratio is affected by two main factors, namely the bandwidth and sensitivity of the detector, and the initial amplitude of the PA emission. The detector can be optimised for its chosen application, but the PA emissions are dictated by the properties of the medium and laser fluence. Tuneable lasers can be used to provide functional images based on different absorption coefficients within the medium, for example determination of the oxygenation level in individual blood vessels in vivo ${ }^{6}$. However, to ensure sufficient contrast to resolve individual vessels $(<350 \mu \mathrm{m})$, these techniques are generally limited to depths of a few millimetres.

The laser fluence is attenuated exponentially with increasing depth where the initial fluence is set through safety guidelines. This ultimately limits the depth at which a PA emission can be detected. This can be improved upon through the use of optical contrast agents, where it has been shown to be possible to generate emissions at depths up to and in excess of $50 \mathrm{~mm}^{7,8,9}$. Plasmonic nanoparticles (NP) can be used as a contrast agent for photoacoustic imaging, since they can be manufactured to be very efficient absorbers at specific wavelengths of light ${ }^{10}$. Gold NPs can be functionalised to target specific cell populations promoting both contrast and ability to delineate tissue types ${ }^{11}$. It has been shown that this technique can be used to image tumour cells with $500 \mu \mathrm{m}$ resolution at depth up to $60 \mathrm{~mm}$, in vitro ${ }^{12}$. The increased optical absorption of laser light can result in an increase in temperature of the NPs sufficient to vaporise the local surrounding medium. Provided the vapour pressure was great enough to overcome the surface tension and viscosity in the medium a vapour bubble will form. This bubble would then grow to a maximum radius, where it would be rapidly cooled and condensed by the surrounding medium resulting in a collapse ${ }^{13}$. Laser-generated vapour bubbles can be used for selective identification of cell populations, through the detection of emitted acoustic signals ${ }^{14}$, and/or optical detection $^{13}$. In addition these bubbles can be used to destroy cells ${ }^{15}$. However, the mechanism for cell destruction, whether it is from thermal and/or mechanical processes, is presently not fully understood. The laser fluence required for bubble generation typically exceeds the maximum permissible exposure, limiting these techniques to in vitro and animal studies ${ }^{16}$, which is further complicated by the fragmentation of the NPs, resulting in poor reproducibility for these studies $^{17}$.

The potential benefits from nanoparticle-targeted vapour bubble formation, from increases in imaging contrast and depth, to cell specificity and destruction, are clear. However, a method for the repeatable generation of bubbles at laser fluences which could be used clinically needs to be developed. In an earlier study ${ }^{18}$, a method for the generation of inertial cavitation through the combination of light and sound was demonstrated. It showed that through the use of this hybrid technique, inertial cavitation could be generated from gold NPs at laser fluences and acoustic pressures much less than when either mechanism was used separately. Inertial cavitation is characterised as a bubble that undergoes a rapid expansion to many times its initial radius and violently collapses ${ }^{19}$. The collapse of a bubble results in the emission of a broadband pressure wave, which is commonly used to identify the presence of inertial cavitation ${ }^{19}$.

Complementing PA applications, NP-nucleated bubbles have a potential application in the enhancement of high intensity focused ultrasound (HIFU), which is a non-invasive modality for the ablation of soft tissue tumours. It is believed that 
the enhanced energy deposition resulting from the presence of inertial cavitation could increase the efficacy of this modality $^{20}$. However, the very high acoustic pressures and the unpredictable nucleation process in many biological tissues limits the potential therapeutic application for inertial cavitation.

This study presents the use of gold nanoparticles simultaneously exposed to light and sound as a nucleation source for inertial cavitation. Through comparisons with the photoacoustic emissions, the broadband emissions generated through inertial collapse (IC) are investigated for their potential benefits for photoacoustic imaging.

\section{EXPERIMENTAL SETUP}

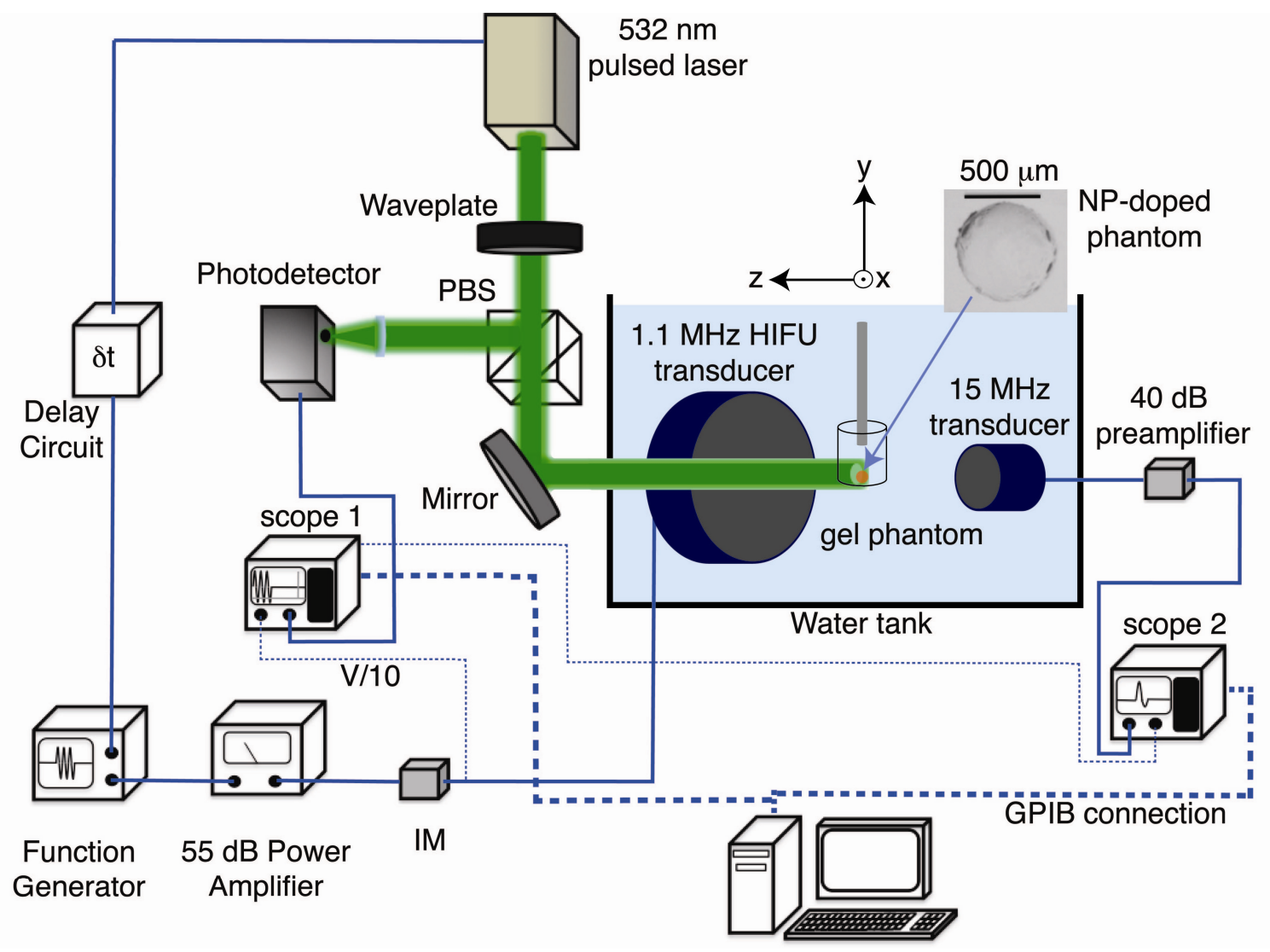

Figure 1. A schematic of the experimental apparatus used in the nanoparticle study, where IM - impedance matching circuit and PBS - polarising beam splitter.

Fig. 1 shows a schematic of the experimental apparatus used for this study. A Nd:YAG pulse laser (Minilite-10, Continuum, wavelength $532 \mathrm{~nm}$, pulse length $10 \mathrm{~ns}$, beamwidth $2.38 \mathrm{~mm}$ ) and a $1.1 \mathrm{MHz}$ single-element, focused piezoelectric HIFU transducer (H101, Sonic Concepts, focal length $63 \mathrm{~mm}, 6 \mathrm{~dB}$ focal region 19x2 mm) were used to expose a polyacrylamide gel sphere to optical and ultrasound fields. The sphere was doped with $80 \mathrm{~nm}$ gold NPs (EM.GC80, British Biocell International) at a concentration of $10^{9}$ particles $/ \mathrm{ml}$. The diameter of the sphere was $500 \mu \mathrm{m}$, and it was embedded in a polyacrylamide gel cylinder $(10 \times 40 \mathrm{~mm})$ that had a mounting screw set in place during manufacture (Fig. 1). A waveplate and polarizing beam-splitter was used to vary the laser fluence on the target over the 
range $0-25.3 \mathrm{~mJ} / \mathrm{cm}^{2}$. This variable beam-splitter was calibrated a priori using an energy meter (J-50MB-HE, FieldMaxII-P, Coherent). The sphere was exposed to this fluence range for the acoustic peak negative pressures of 0,1 and 1.5 MPa. The HIFU transducer was calibrated using a membrane hydrophone (Precision Acoustics) in water. As the laser 'Lamp Sync' TTL trigger occurred $150 \mu$ s before the light flash, a custom built variable delay circuit was used to trigger the 3-cycle acoustic pulse $42.5 \pm 0.02 \mu$ s before the light flash, to allow for the third cycle rarefaction pressure to be incident on the target simultaneously with the laser light. The timing was monitored through the use of a photodetector (1607, New Focus) and a voltage probe, which were recorded on an oscilloscope (LT344L, LeCroy), 'scope 1' in Fig. 1, and downloaded to a computer after each laser shot. A $15 \mathrm{MHz}$ focused broadband transducer (V313, Olympus NDT inc, focal length $19 \mathrm{~mm}, 6 \mathrm{~dB}$ focal region $6.4 \times 0.3 \mathrm{~mm}$ ) was used passively to detect the PA and inertial collapse (IC) emissions generated by the thermal expansion of the NPs, and bubble collapse. These signals were then amplified (5185 Wideband Preamplifier, Signal Recovery, gain $40 \mathrm{~dB}$ ) and recorded on an oscilloscope (LT342L, LeCroy), 'scope 2' in Fig. 1, which was then downloaded to a computer after each shot.

Alignment of the laser with the HIFU and detection transducer was achieved by using a $2 \mathrm{~mm}$ ball bearing as a scattering target. An alignment target was mounted in a separate gel cylinder and positioned centrally in the laser beam. The HIFU and detection transducers were mounted on three-axis micrometer stages and aligned using pulse-echo and -receive techniques, thus ensuring the laser pulse and acoustic foci were co-aligned to a fixed region. Locating the NP-doped sphere into this region was achieved by positioning with computer controlled micrometer stages, such that the maximum PA emission was detected, from light alone at a fluence of $25.3 \mathrm{~mJ} / \mathrm{cm}^{2}$.

\section{RESULTS AND DISCUSSION}

(a)

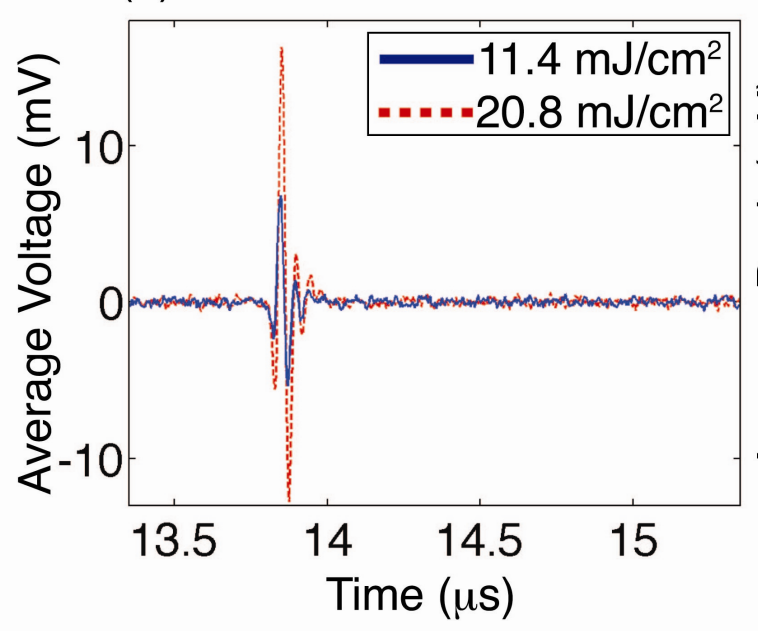

(b)

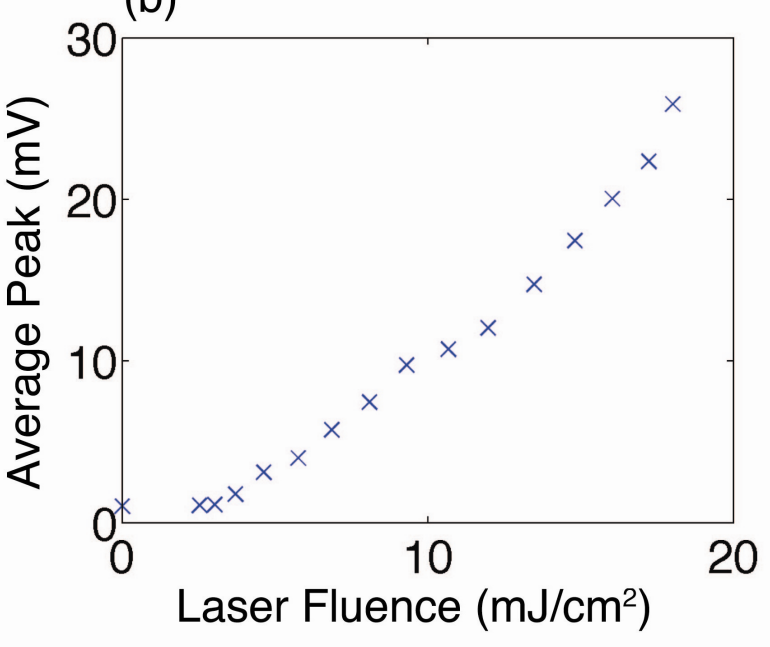

Figure 2. (a) The averaged photoacoustic emission from 500 repeat shots, for a laser fluence of 11.4 (blue line) and $20.8 \mathrm{~mJ} / \mathrm{cm}^{2}$ (dashed red line). (b) Peak averaged photoacoustic emission as a function of incident laser fluence. 
Fig. 2(a) gives two examples of the detected PA emission at two laser fluences averaged for 500 repeat shots in the NPdoped sphere; No HIFU field was present during these exposures. The averaged peak amplitude of the PA emissions at the two laser fluences was 6.46 and $16.27 \mathrm{mV}$ respectively. For the higher laser fluence, the average peak amplitude was approximately twice, which was consistent with the approximate doubling in the incident laser fluence. Fig. 2(b) shows the averaged peak PA emission as a function of laser fluence. For fluences up to and including $4.26 \mathrm{~mJ} / \mathrm{cm}^{2}$ the PA emissions were not detectible above the noise level of the system. After this point, the peak value increases approximately linearly with increasing laser fluence.
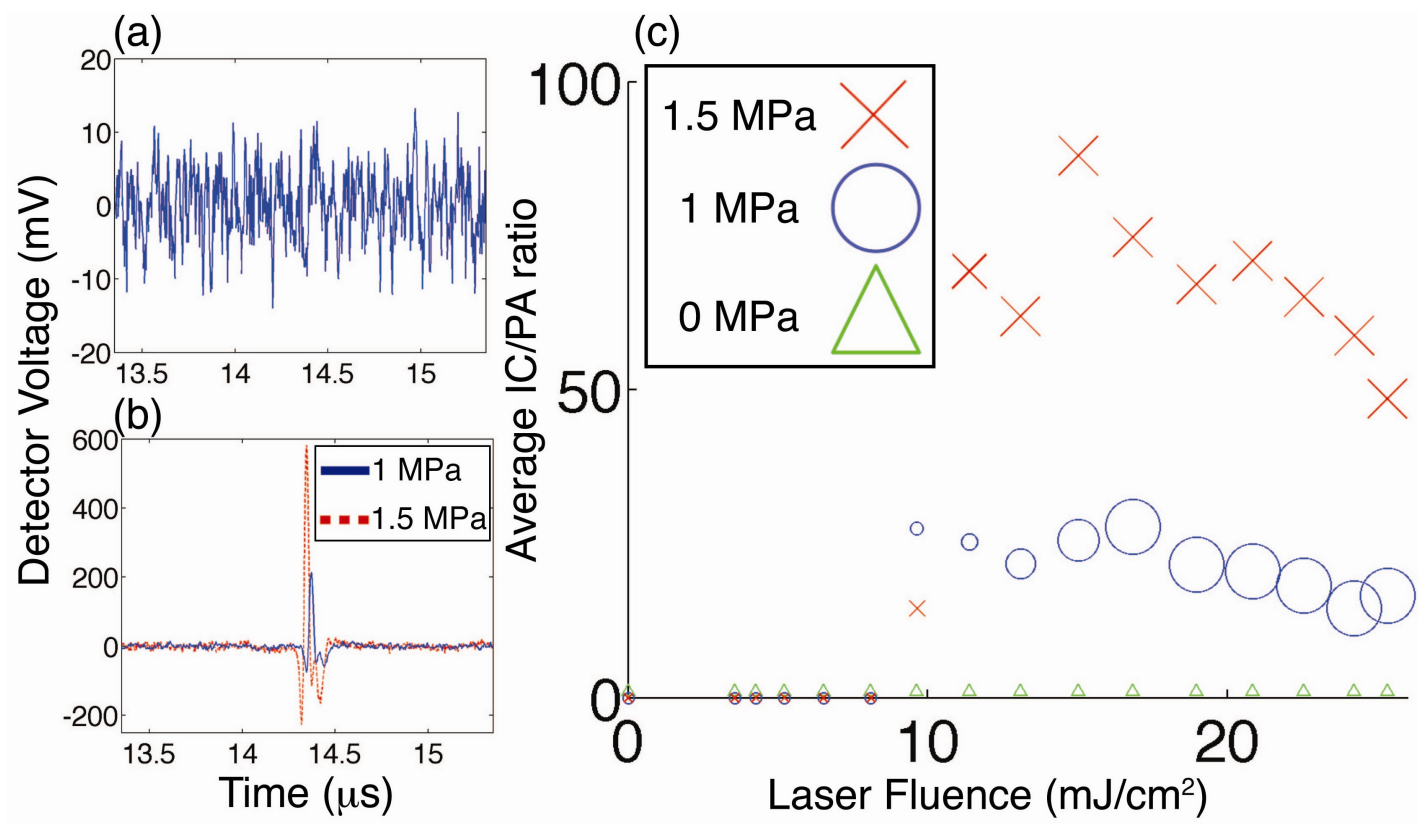

Figure 3. (a). A single measurement at a fluence of $11.4 \mathrm{~mJ} / \mathrm{cm}^{2}$ with the HIFU peak negative pressure of $0 \mathrm{MPa}$. (b) Two broadband, inertial collapse (IC) emissions generated from inertial cavitation from a single exposure with a laser fluence of $11.4 \mathrm{~mJ} / \mathrm{cm}^{2}$ and peak negative pressure of 1 (blue line) and 1.5 MPa (dashed red line). (c) The averaged peak inertial collapse/photoacoustic ratio, for a laser fluence range of $0-25.3 \mathrm{~mJ} / \mathrm{cm}^{2}$ and peak negative pressures of 0 (green triangle), 1.0 (blue circle) and 1.5 MPa (red cross). Marker size represents the probability of an inertial collapse occurring, where $0 \%$ is the smallest and $100 \%$ the largest.

Fig. 3(a) shows that without averaging, at this laser fluence $\left(11.4 \mathrm{~mJ} / \mathrm{cm}^{2}\right)$, the PA emission detected from the illumination of the $500 \mu \mathrm{m}$ NP-doped sphere is indistinguishable from the noise level of this system. However, through the application of a HIFU field to the same target, with a peak negative pressure of 1 or $1.5 \mathrm{MPa}$, secondary emission can be generated (Fig. 3b). This emission is resultant from collapse of inertial cavitation in the NP-doped sphere, which occurs approximately $0.5 \mu \mathrm{s}$ after the PA emissions (Fig. 2a). This delay corresponds to the presence of the compression cycle of the acoustic wave in the sphere. As the experiment was designed such that the diameter of the doped spheres was smaller than half a wavelength at the frequency used $\left(\approx 700 \mu \mathrm{m}\right.$, for a gel phantom at $\left.20^{\circ} \mathrm{C}\right)$, this effect only occurs in the interaction region between the light and acoustic fields. A 3-cycle HIFU pulse ensured that the transducer had reached optimum operating conditions. However, a single cycle of sufficient pressure amplitude would be adequate, 
assuming that output of the transducer was such that the rarefaction cycle was incident on the target before the compression cycle. The arrival times for these two emissions differ by approximately $20 \mathrm{~ns}$, which in this case is due to the timing uncertainty in the delayed trigger system. For the representative IC emissions shown in Fig. 3(b), peak amplitudes of 208 and $582 \mathrm{mV}$ were measured for a pressure of 1 and $1.5 \mathrm{MPa}$. The amplitude of these single IC emissions are approximately 32 and 90 times larger than the peak averaged PA emission, for the same laser fluence. This highlights the potential gain in contrast and subsequently imaging depth that these emissions could provide for imaging applications. The ratio for the peak averaged IC to the peak averaged PA for the phantom over all exposure parameters is shown in Fig. 3(c). Probability of an IC event is represented by the marker size, where the smallest are $0 \%$ and the largest are $100 \%$. An IC event was said to have occurred if the detected signal exceeded the threshold level of $50 \mathrm{mV}$ within $\pm 0.4 \mu$ s of the expected arrival time of the IC. This figure shows that the ratio is greatest for the smallest laser fluences, but has the lowest probability of an IC emission occurring. The reason for this maximum ratio is the amplitude of the PA emission increases linearly with fluence (Fig. 2b), whereas the amplitude of the IC remains approximately constant with a fixed acoustic pressure. Since the amplitude of the IC emissions were dictated by the acoustic pressure amplitude, the fluence would only need to be sufficient to nucleate inertial cavitation from the NPs. Since the tissue penetration of ultrasound is significantly greater than light, this technique could be used to dramatically increase the signal-to-noise ratio of the broadband emissions generated from gold nanoparticles.

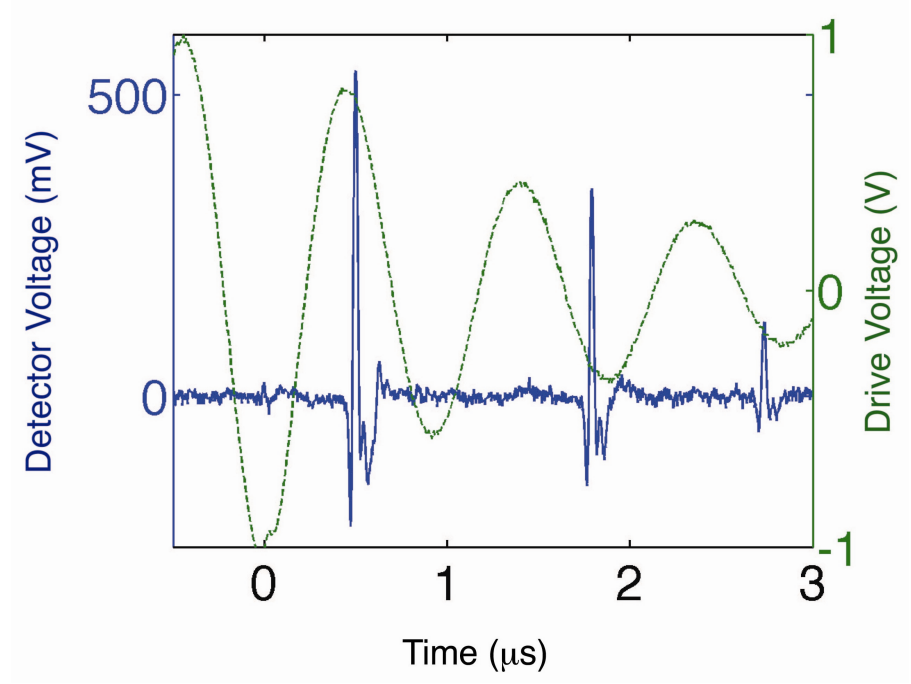

Figure 4. A plot showing a series of IC emissions (blue line) from a single exposure at a fluence of $15.0 \mathrm{~mJ} / \mathrm{cm}^{2}$ and pressure of 1.5 MPa, compared with the measured drive voltage (dashed green line). The time scale on the plot has been set such that $t_{0}$ is equal to the time of the laser flash.

Fig. 4 shows a PA emission and multiple IC emissions for a single exposure, at a fluence of $15.0 \mathrm{~mJ} / \mathrm{cm}^{2}$ and a peak negative pressure of $1.5 \mathrm{MPa}$, compared with the measured drive voltage to the HIFU transducer. The time scale in this figure has been set that $t_{0}$ represents the time at which the light and sound was coincident on the doped sphere, thus neglecting the time of flight for each of these measurements. The particular example shows a series of IC emissions that occur after the presence of a compression cycle due to the 'ring down' in the output of the HIFU transducer. As the amplitude of the drive voltage (and subsequently the pressure field) decays, the amplitude of the IC also decays. This 
demonstrates that from a single laser pulse it was possible to generate multiple bubble growth and collapse cycles, which respond directly to the incident acoustic field. This highlights the potential benefits of this technique for the controlled and repeatable generation of inertial cavitation.

(a)

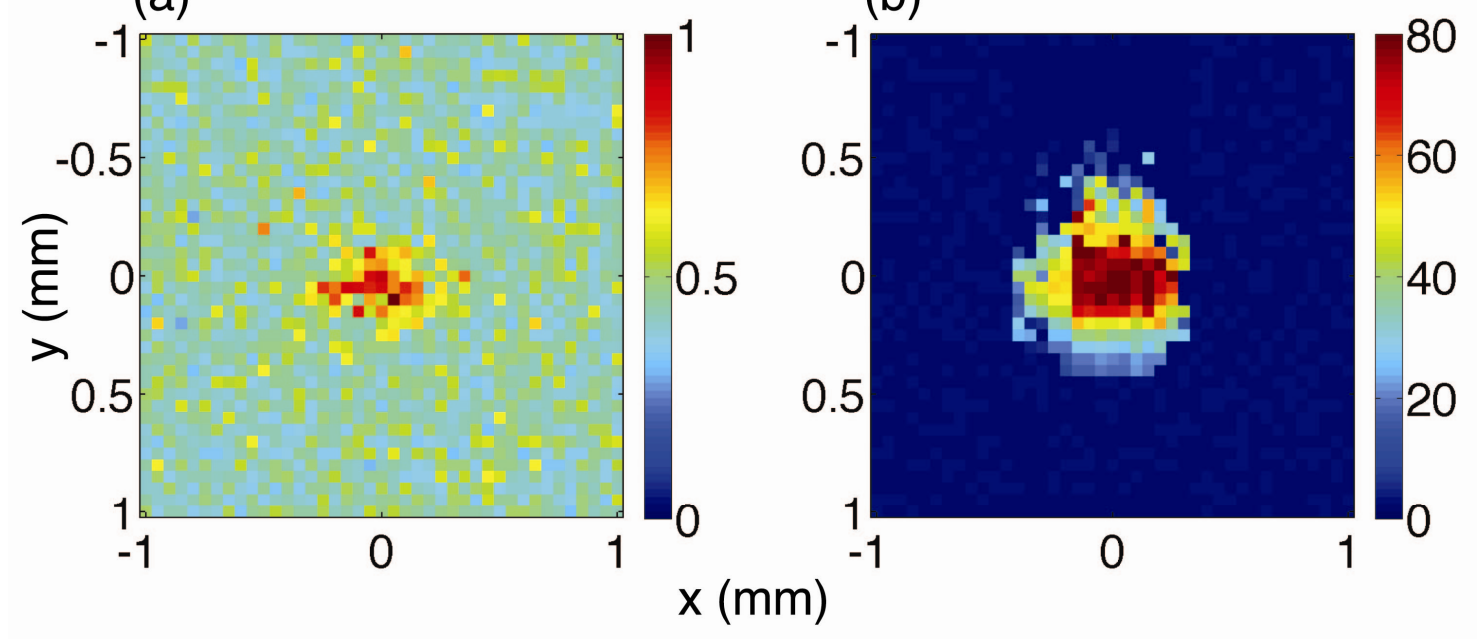

Figure 5. Two raster scans of the nanoparticle-doped sphere (diameter $500 \mu \mathrm{m}$ ). (a) The peak averaged $(n=10)$ photoacoustic emission for a fluence of $11.4 \mathrm{~mJ} / \mathrm{cm}^{2}$ and (b) the peak inertial collapse emission generated at the same fluence but with the addition of a 1.5 MPa HIFU field. The colour scale in each image has been normalised to the peak signal in (a).

Fig. 5 shows images generated from two separate raster scans of the NP-doped sphere, where (a) is generated from the photoacoustic and (b) the inertial collapse emissions. The image shown in Fig. 5(a) was generated by scanning the doped sphere through the light and sound field at steps of $50 \mu \mathrm{m}$, where at each position 10 repeat shots were taken and the waveforms recorded. The averaged peak of the detected PA emission occurring within a $\pm 0.6 \mu$ s window of the expected arrival time of these emissions for a laser fluence of $11.4 \mathrm{~mJ} / \mathrm{cm}^{2}$ was assigned at each pixel location. Averaging over the repeat measurements at each location was necessary to ensure that the peak PA emission was detectable above the noise level. Fig. 5(b) shows the raster scan of the same doped region and laser fluence with the addition of a $1.5 \mathrm{MPa}$ HIFU field. The image was generated with the same method as that used for the PA image, but without averaging over the repeat measurements. IC emissions were significantly greater than the noise level, removing the need for averaging. This figure demonstrates that just through the application of an acoustic field, a dramatic increase, in this case 80 times, in the detected emissions from the illuminated nanoparticles can be achieved. The generation of the IC emissions was only possible when a rarefaction cycle was coincident with the laser pulse. For example, if the arrival phase was shifted such that the compression cycle was coincident with the laser pulse, no IC emissions were generated. This could be advantageous when working in a light diffuse medium with an unknown concentration of nanoparticles since it would only be in this interaction region that emissions would be generated.

The same sample was repeatedly exposed to both light and sound without any evidence that the nanoparticles were being fragmented. This suggests that this technique could also have a particular advantage when compared with laser vapour 
bubble generation, in that they can be generated at clinically useful fluence and pressure levels and be repeatedly exposed. It should be noted that no IC emissions were generated from the use of either a 1 or $1.5 \mathrm{MPa} 3$-cycle HIFU pulse in these NP-doped spheres, and that only PA emissions were detected over the fluence range used.

\section{CONCLUSIONS}

This study demonstrated the potential use of gold nanoparticles to nucleate inertial cavitation only in the presence of both laser light and an ultrasound field. The detected amplitude of the acoustic emissions generated from the collapse of inertial cavitation was up to two orders of magnitude greater than the PA emissions for the same fluence levels. This highlights a potential use of NP in this manner as a 'photoacoustic contrast agent'. Repeat exposures were conducted in the same nanoparticle-doped sphere without any evidence of fragmentation in the nanoparticles. This is a significant advantage over current techniques for the generation of vapour bubbles, which could also be applied to the use of bubbles for cell targeting and destruction at clinically useful exposure parameters. Multiple inertial collapse emissions could be generated from single laser shots where the amplitude of the emissions were governed by the acoustic paramaters, demonstrating the generation of controllable inertial cavitation in a HIFU field. This could have potential applications for the enhancement of HIFU treatments through the controlled and repeatable generation of inertial cavitation.

\section{ACKNOWLEDGEMENT}

This research was supported by the Bernard M. Gordon Center for Subsurface Sensing and imaging Systems under the National Science Foundation (EEC-9986821) and a Boston University Dean’s Catalyst Award.

\section{REFERENCES}

[1] Xu, M. and Wang L. H., "Photoacoustic imaging in biomedicine," Review of Scientific Instruments 77(4), 041101$22(2006)$.

[2] Karabutov, A.A., Oraevsky, A.A, [Time-Resolved Detection of Optoacoustic Profiles for Measurement of Optical Energy Distribution in Tissues], in Handbook of Biomedical Diagnostics, V. V. Tuchin, Editor. SPIE press: Bellingham, Washington USA. 585-650 (2002).

[3] Wang, X., Xie, X., Ku, G. and Wang, L. V., "Noninvasive imaging of hemoglobin concentration and oxygenation in the rat brain using high-resolution photoacoustic tomography," Journal of Biomedical Optics 11(2), 024015-9 (2006).

[4] Zhang, H. F., Maslov, K., Stoica, G. and Wang, L. V., "Functional photoacoustic microscopy for high-resolution and noninvasive in vivo imaging," Nat Biotech 24(7), 848-851 (2006).

[5] Andreev, V. G., Karabutov, A. A. and Oraevsky A. A., "Detection of ultrawide-band ultrasound pulses in optoacoustic tomography," IEEE Transactions on Ultrasonics Ferroelectrics and Frequency Control 50(10), 13831390 (2003).

[6] Zhang, H. F., Maslov, K., Li. M., Stoica, G. and Wang, L. V., "In vivo volumetric imaging of subcutaneous microvasculature by photoacoustic microscopy,” Opt. Express 14(20), 9317-9323 (2006). 
[7] Ermilov, S. A., Khamapirad, T., Conjusteau, A., Leonard, M. H., Lacewell, R., Mehta, K., Miller, T. and Oraevsky, A. A., "Laser optoacoustic imaging system for detection of breast cancer," Journal of Biomedical Optics 14(2), 024007 (2009).

[8] $\mathrm{Ku}, \mathrm{G}$. and Wang L.V., "Deeply penetrating photoacoustic tomography in biological tissues enhanced with an optical contrast agent," Opt. Lett. 30(5), 507-509 (2005).

[9] Funke, A.R., Aubry, J., Fink, M., Boccara, A. and Bossy, E., "Photoacoustic guidance of high intensity focused ultrasound with selective optical contrasts and time-reversal," Applied Physics Letters 94(5), 054102-3 (2009).

[10] Yang, X., Stein E.W., Ashkenazi S., and Wang L. V., "Nanoparticles for photoacoustic imaging," Wiley Interdisciplinary Reviews: Nanomedicine and Nanobiotechnology 1(4), 360-368 (2009).

[11] Mallidi, S., Larson, T., Aaron, J., Sokolov, K. and Emelianov, S. "Molecular specific optoacoustic imaging with plasmonic nanoparticles," Opt. Express 15(11), 6583-6588 (2007).

[12] Copland, J.A., Eghtedari, M., Popov, V. L., Kotov, N., Mamedova, N., Motamedi, M. and Oraevsky, A. A., "Bioconjugated gold nanoparticles as a molecular based contrast agent: implications for imaging of deep tumors using optoacoustic tomography,” Molecular Imaging and Biology 6(5), 341-349 (2004).

[13] Lapotko, D., "Optical excitation and detection of vapor bubbles around plasmonic nanoparticles," Opt. Express 17(4), 2538-2556 (2009).

[14] Egerev, S., Ermilov, S., Ovchinnikov, O., Fokin, A., Guzatov, D., Klimov, V., Kanavin, A. and Oraevsky A., "Acoustic signals generated by laser-irradiated metal nanoparticles," Appl. Opt. 48(7), C38-C45 (2009).

[15] Zharov, V. P., Mercer, K. E., Galitovskaya, E. N. and Smeltzer, M. S., "Photothermal Nanotherapeutics and Nanodiagnosics for Selective Killing of Bacteria targeted with Gold Nanoparticles,” Biophysical Journal 90(2), 619-627 (2006).

[16] Hleb, E. Y., Hafner J. H., Myers J. N., Hanna E. Y., Rostro B. C., Zhdanok S. A. and Lapotko D.O., "LANTCET: elimination of solid tumor cells with photothermal bubbles generated around clusters of gold nanoparticles," Nanomedicine 3(5), 647-667 (2008).

[17] Lapotko, D., "Plasmonic nanoparticle-generated photothermal bubbles and their biomedical applications," Nanomedicine 4(7), 813-845 (2009).

[18] Farny, C. H., Wu, T., Holt, R. G., Murray, T. W., and Roy, R. A., "Nucleating cavitation from laser-illuminated nano-particles,” Acoustics Research Letters Online 6(3), 138-143 (2005).

[19] Leighton, T.G., [The Acoustic Bubble], 1st ed. Academic Press, London (1994).

[20] Haar, G. t. and Coussios C., "High Intensity Focused Ultrasound: Past, present and future," International Journal of Hyperthermia 23(2), 85-87 (2007). 